\begin{tabular}{|c|l|}
\hline Title & $\begin{array}{l}\text { Generation of Radical Species from Cyclohexane-1,2-dione and the Reaction with Olefins: Preparation of 4,5-Dihydro- } \\
7(6 \mathrm{H}) \text {-benzofuranone Derivatives }\end{array}$ \\
\hline Author(s) & Miura, Masanori; A rai, Noriy oshi; Narasaka, Koichi \\
\hline Citation & $\begin{array}{l}\text { Bulletin of the Chemical Society of Japan, 71(6), 1437-1441 } \\
\text { https://doi.org/10.1246/0csj.71.1437 }\end{array}$ \\
\hline Issue Date & 1998 \\
\hline Doc URL & http://hdl.handle.net/2115/70768 \\
\hline Type & article \\
\hline File Information & bcs..71.1437.pdf \\
\hline
\end{tabular}

Instructions for use 


\title{
Generation of Radical Species from Cyclohexane-1,2-dione and the Reaction with Olefins: Preparation of 4,5-Dihydro-7(6H)-benzofuranone Derivatives
}

\author{
Masanori Miura, Noriyoshi Arai, and Koichi Narasaka* \\ Department of Chemistry, School of Science, The University of Tokyo, 7-3-1, Hongo, Bunkyo-ku, Tokyo 113-0033
}

(Received January 16, 1998)

Oxidation of cyclohexane-1,2-dione with ammonium hexanitratocerate(IV) (CAN) generates 2,3-dioxocyclohexyl radical, which reacts with electron-rich olefins to afford the corresponding addition products. The adducts thus generated are converted to 4,5-dihydro-7 $(6 H)$-benzofuranone by acid treatment. In addition to cyclohexane-1,2-dione, radical species are also generated from cyclopentane-1,2-dione and cycloheptane-1,2-dione.

Though 4,5-dihydro-7(6H)-benzofuranone derivatives are considered to be useful synthetic intermediates and are found in natural products, such as mascarol ${ }^{\text {1a) }}$ and aoifuranone, ${ }^{1 \mathrm{~b})}$ few methods have been known for the preparation of 4,5-dihydro-7 $6 H$ )-benzofuranone skeleton. Walsh and Stone synthesized the skeleton using the intramolecular Friedel-Crafts acylation of 4-(3-furyl)butanoyl chloride, which was prepared through several steps from 3-furaldehyde. ${ }^{2 a)}$ Corey and Xiang reported the conversion of 4,5,6,7-tetrahydrobenzofurans to the 7-oxo derivatives by the stepwise oxidation of the 7-position. ${ }^{2 b)}$ It is expected that 3-(2-oxoalkyl)cyclohexane1,2-dione 2 could be converted to 4,5-dihydro-7 $(6 H)$-benzofuranone 3 by acid-catalyzed condensation. The triketone 2 is, however, not readily prepared from cyclohexane-1,2dione (1) because the alkylation at the 3-position of $\mathbf{1}$ does not proceed due to the poor nucleophilicity of its enolate (Eq. 1). ${ }^{3)}$ Even the dianion of $\mathbf{1}$ could be alkylated only with allyl and 2-propynyl bromides to give the corresponding 3substituted products in moderate yield. ${ }^{4)}$

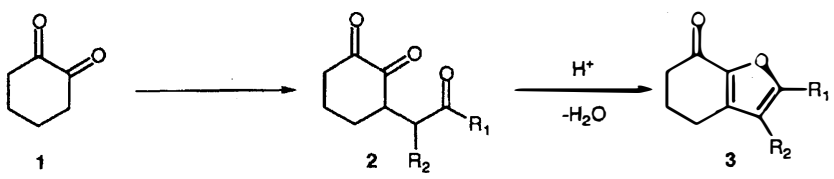

We have studied the generation of radical species by oneelectron oxidation with metallic oxidants and their intermolecular addition reactions to olefins, ${ }^{5 \mathrm{a}-\mathrm{f})}$ and intended to apply this radical process to the alkylation of cyclohexane1,2-dione (1). 1,3-Dicarbonyl compounds, which have large enol contents, are known to be oxidized with metallic oxidants to generate their 2-yl radicals, which give the addition products with olefinic compounds. ${ }^{67)}$ Since cyclohexane-1, 2-dione (1) exists mostly in its enol form, ${ }^{8)}$ one electron oxidation of 1 with metallic oxidants would give the 3 -yl radical, which could be employed to introduce the substituent at the 3-position of $\mathbf{1}$.
Based on this assumption, generation of the 3-yl radical of 1 was examined and this method was applied to the preparation of 4,5-dihydro-7(6H)-benzofuranone derivatives.

\section{Results and Discussion}

Generation of 2,3-Dioxocyclohexyl Radical and the Addition Reaction to Trimethyl(1-phenylvinyloxy)silane. Oxidation of cyclohexane-1,2-dione (1) was tried with some metallic oxidants in the presence of 2 molar amounts of trimethyl(1-phenylvinyloxy)silane (4a) as the radical acceptor. Ammonium hexanitratocerate (CAN) (2.1 molar amounts) oxidized 1 smoothly in acetonitrile and 3-(2-oxo-2-phenylethyl)cyclohexane-1,2-dione (2a) was obtained in $83 \%$ yield, while the oxidation did not occur with ferrocenium hexafluorophosphate ${ }^{9)}$ or manganese(III) 2-pyridinecarboxylate. ${ }^{10)}$ The yield of the triketone $\mathbf{2 a}$ was affected by the molar ratio of $\mathbf{1}$ to $\mathbf{4 a}$, but was not much affected by the reaction temperature (Table 1). That is, in the presence of 3 molar amounts of the silyl vinyl ether $\mathbf{4 a}$, the triketone $\mathbf{2 a}$ was obtained in $89 \%$ yield, whereas the yield decreased to $52 \%$ with 1.5 molar amounts of $\mathbf{4 a}$ (Entries $1-3$ ). Acetonitrile was found to be a suitable solvent. As mentioned above,

Table 1. Effect of Temperature and Amount of Trimethyl(1-phenylvinyloxy)silane (4a) on the Reaction of 1 with CAN

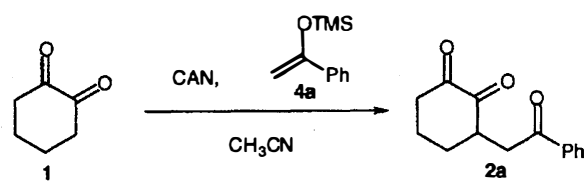

\begin{tabular}{cccc}
\hline Entry & Temp $/{ }^{\circ} \mathrm{C}$ & Molar ratio of 4a to 1 & Yield/\% \\
\hline 1 & -35 & 3.0 & 89 \\
2 & -35 & 2.0 & 83 \\
3 & -35 & 1.5 & 52 \\
4 & -45 & 3.0 & 89 \\
5 & 0 & 3.0 & 88 \\
\hline
\end{tabular}


the triketone $2 \mathbf{a}$ was obtained in $89 \%$ yield by the reaction in acetonitrile at $-35^{\circ} \mathrm{C}$, while the reactions in $N, N$-dimethylformamide (DMF) or methanol gave the product $2 \mathbf{a}$ in 59 and $74 \%$ yield, respectively, along with a small amount of 1 , 4-diphenylbutane-1,4-dione, a self-coupling product of the silyl vinyl ether $\mathbf{4 a}$. This reaction is considered to proceed as depicted in the Scheme 1. Due to the dipole repulsion of the two carbonyl groups, cyclohexane-1,2-dione (1) exists mostly in its enol form A ${ }^{8)}$ This large enol content is essential for the success of the oxidation. In fact, an acyclic 1,2diketone, 1-phenylpropane-1,2-dione, could not be oxidized with CAN under the similar reaction conditions. The enol form $\mathbf{A}$ is oxidized with CAN to generated the 3-yl-radical $\mathbf{B}$, which adds to the silyl vinyl ether $\mathbf{4 a}$, giving an addition intermediate $\mathbf{C}$. By the successive oxidation, $\mathbf{C}$ is converted to cation $\mathrm{D}$, from which the silyl group is eliminated to afford the triketone $\mathbf{2 a}$.

Conversion of 3-(2-Oxoalkyl)cyclohexane-1,2-dione Derivatives to 4,5-Dihydro-7(6H)-benzofuranone Derivatives. In the above-mentioned reaction, the triketone $\mathbf{2 a}$ was isolated by extraction followed by the evaporation of the solvent and acetophenone, which resulted from the hydrolysis of 4a. Interestingly, 2a was isolated completely as the keto form and not isomerized into the enol form on standing at room temperature. The keto form, however, isomerized into the enol form when the crude $\mathbf{2 a}$ was chromatographed on Silica gel or on activated alumina, and the enol form of 2a was adsorbed partially on the adsorbents. This made the further purification of $\mathbf{2 a}$ difficult. The isolation of $\mathbf{2 a}$ is, however, not necessary for the preparation of 4,5-dihydro$7(6 H)$-benzofuranone 3a from 1 . That is, the reaction of 1 and $\mathbf{4 a}$ was quenched with aqueous sodium thiosulfate; then the crude mixture was treated with Amberlyst 15 to afford 2phenyl-4,5-dihydro-7(6H)-benzofuranone (3a) in 78\% yield (Eq. 2). Camphorsulfonic acid did not promote the condensation, and 3a was isolated in 59\% yield by the use of $p$ toluenesulfonic acid.

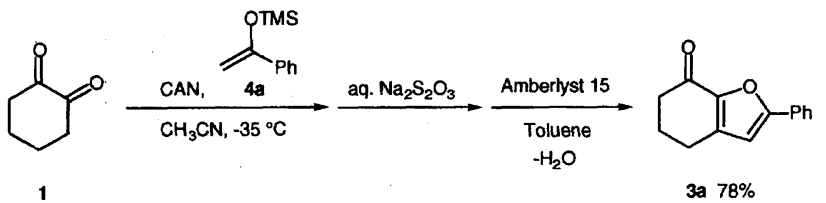

Various 4,5-dihydro-7(6H)-benzofuranone derivatives were synthesized by this method (Table 2). Silyl 1-substituted vinyl ethers $\mathbf{4 a}-\mathbf{e}$ reacted with $\mathbf{1}$ to afford 4,5-dihydro$7(6 H)$-benzofuranones 3a-e in moderate yield (Entries 1 5). 2-Non-substituted 4,5-dihydro-7 $(6 H)$-benzofuranone $3 \mathbf{f}$ was prepared in $49 \%$ yield by employing 20 molar amounts of (1-ethoxyvinyl)trimethylsilane (4f), after the treatment of the crude product with tetrabutylammonium fluoride (Entry $6)$. In addition to silyl vinyl ethers, the reactions with methyl 1-phenylvinyl ether (4g) and 2-phenylpropene (4h) also produced the products $\mathbf{3 a}$ and $\mathbf{3 h}$, respectively, in good yields (Entries 7 and 8). The hemiacetal-type product $\mathbf{3 h}$ is generated by the hydration of 2-methyl-2-phenyl-2,3,4, 5-tetrahydro-7 $6 H)$-benzofuranone during isolation. In fact, 2-methyl-2-phenyl-2,3,4,5-tetrahydro-7 $(6 H)$-benzofuranone which was obtained as a side product (5\% yield) in the Entry 8 was found to be readily hydrated on Silica gel to $\mathbf{3 h}$. Propiononitrile was used as co-solvent when the reaction was carried out at $-78{ }^{\circ} \mathrm{C}$ (Entry 2) and when the silyl vinyl ethers $\mathbf{4 d}$ and $\mathbf{4 e}$ were insoluble in acetonitrile (Entries 4 and 5). The reactions of alkyl vinyl ethers in methanol gave the corresponding triketones as the intermediates which were converted to the 4,5-dihydro-7(6H)-benzofuranones $\mathbf{3 f}$ and 3a in 29 and $71 \%$ yield, respectively (Entries 6 and 7), while complex mixtures were given in acetonitrile. Though the reason why only methanol afforded good results is not clearly understood, methanol traps the cation intermediate, which might prevent unfavorable side reactions. Vinyl acetate, allyltrimethylsilane and allyltributylstannane could not be employed successfully as radical acceptors and complex reaction mixtures were obtained.

Generation of 2,3-Dioxocyclopentyl and 2,3-Dioxocycloheptyl Radicals. It is noteworthy that radical species can be also generated from cyclopentane-1,2-dione (5) and cycloheptane-1,2-dione (6) by the oxidation with CAN. The reactions of the cycloalkane-1,2-diones 5 and 6 with the silyl vinyl ether $\mathbf{4 a}$ proceeded to afford triketones $\mathbf{7}$ and $\mathbf{8}$ in good yield, which also exist mostly in their keto forms. By the treatment of the crude products with Amberlyst 15, the triketone $\mathbf{8}$ was cyclized to the corresponding furanone $\mathbf{1 0}$, while 7 did not cyclize at all (Eq. 3).

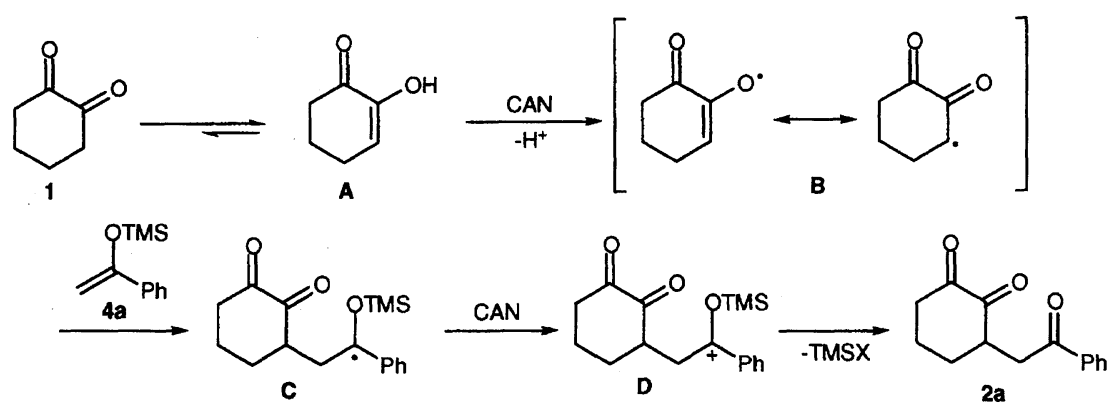

Scheme 1. Mechanism for the addition reaction of the cyclohexane-1,2-dione (1) to the silyl vinyl ether $4 \mathbf{a}$. 
Table 2. Reaction of $\mathbf{1}$ with Various Olefins

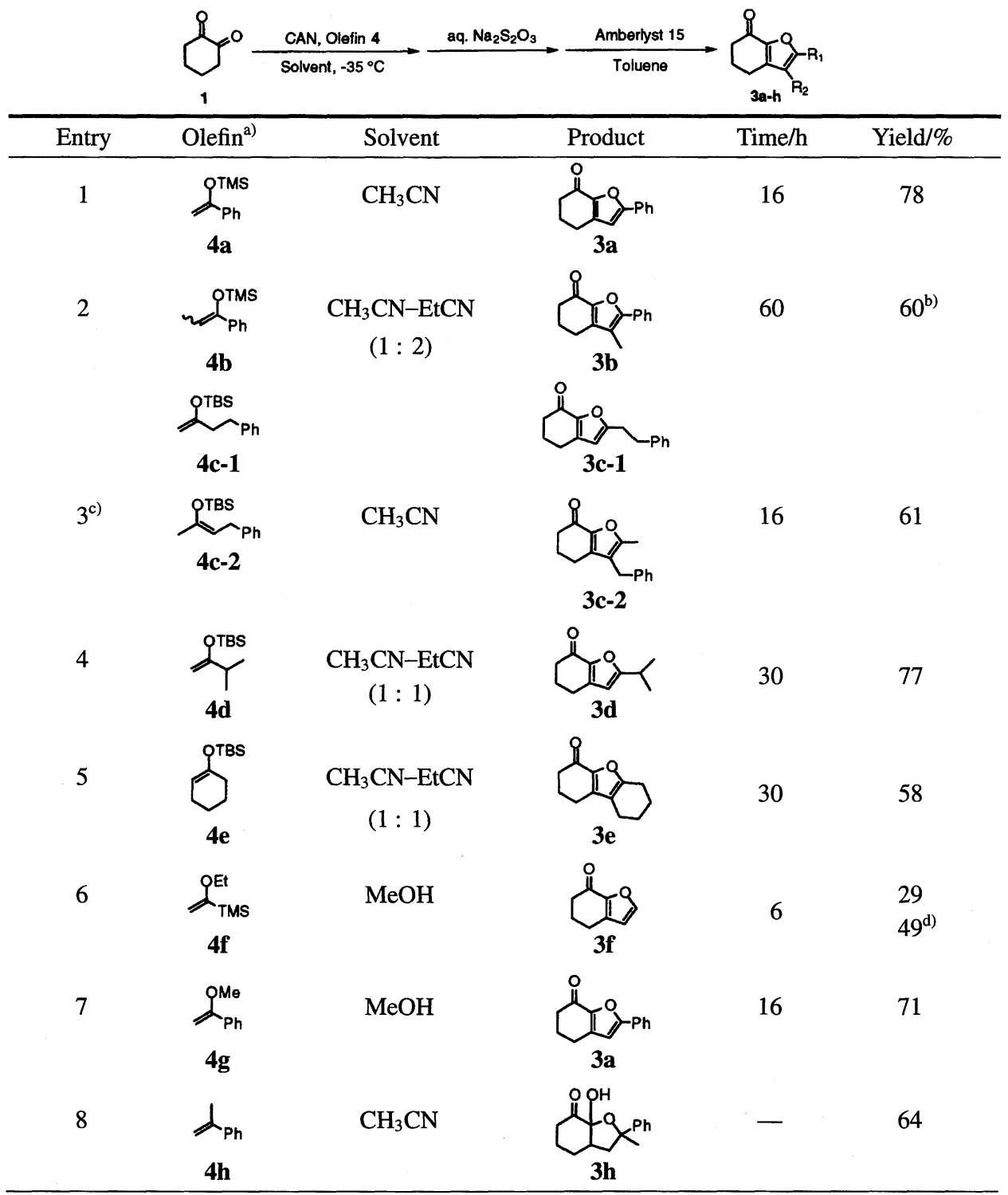

a) 3 molar amounts of olefins were employed. b) The reaction temperature was $-78^{\circ} \mathrm{C}$.

c) $4 c-1: 4 c-2=8: 2$ and $3 c-1: 3 c-2=7: 3$. d) 20 molar amounts of $4 \mathbf{f}$ was employed.
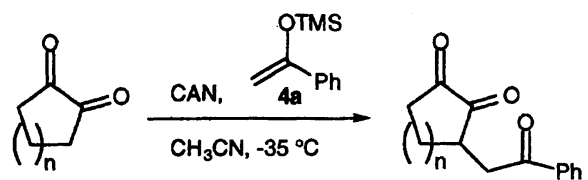

$\begin{array}{rr}n=1 & 5 \\ 3 & 6\end{array}$ $\begin{array}{rrr}n=1 & 7 & 87 \% \\ 3 & 8 & 78 \%\end{array}$

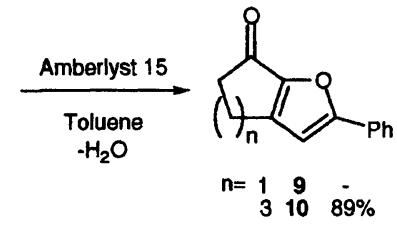

In conclusion, by applying a radical reaction, various carbon-chains can be introduced at the 3-position of cycloalkane-1,2-diones. This process is successfully applied for the preparation of 4,5-dihydro-7(6H)-benzofuranone derivatives.

\section{Experimental}

General. ${ }^{1} \mathrm{H}$ NMR (500 MHz) and ${ }^{13} \mathrm{C} \mathrm{NMR}(125 \mathrm{MHz})$ spectra were recorded on a Bruker AM 500 spectrometer in $\mathrm{CDCl}_{3}$ and $\mathrm{CD}_{2} \mathrm{Cl}_{2}$ solutions. IR spectra were recorded on a Horiba FT 300$\mathrm{S}$ spectrophotometer. High-resolution mass spectra were obtained with a JEOL JMS-SX102A mass spectrometer at an ionization energy of $70 \mathrm{eV}$. The melting points were uncorrected. Elemental analyses were carried out at The Elemental Analysis Laboratory, Department of Chemistry, Faculty of Science, The University of Tokyo. Acetonitrile and propiononitrile were distilled from $\mathrm{P}_{2} \mathrm{O}_{5}$, then from $\mathrm{CaH}_{2}$, and dried over Molecular sieves 4A. Methanol was distilled from magnesium methoxide and dried over Molecular sieves 3A. Toluene was dried over $\mathrm{CaCl}_{2}$ and distilled. CAN (Kanto Chemical Co., Inc., guaranteed grade) was dried under vac- 
uum at $80{ }^{\circ} \mathrm{C}$ for $20-24 \mathrm{~h}$ before use. Cyclopentane-1,2-dione, ${ }^{8)}$ cycloheptane-1,2-dione, ${ }^{11)}$ silyl vinyl ethers $4 \mathbf{a}-\mathbf{e},{ }^{12)}$ (1-ethoxyvinyl)silane (4f), ${ }^{13)}$ and methyl 1-phenylvinyl ether $(\mathbf{4 g})^{14)}$ were prepared after the literature procedures. Activated Alumina (Wako Pure Chemical Industries, Ltd. activated about 300 mesh) was used for column chromatography. Preparative TLC was performed on a silica gel (Wakogel B-5F).

3-(2-Oxo-2-phenylethyl)cyclohexane-1,2-dione (2a). To a solution of CAN $(1.153 \mathrm{~g}, 2.10 \mathrm{mmol})$ in acetonitrile $(5.0 \mathrm{ml})$ was added a solution of trimethyl(1-phenylvinyloxy)silane (4a) (574.5 mg, $2.99 \mathrm{mmol})$ and cyclohexane-1,2-dione (1) $(111.0 \mathrm{mg}$, $0.990 \mathrm{mmol})$ in acetonitrile $(5.0 \mathrm{ml})$ at $-35{ }^{\circ} \mathrm{C}$ under an argon atmosphere. After $15 \mathrm{~min}$, the reaction was quenched by adding 0.1 mol dm ${ }^{-3}$ aq $\mathrm{Na}_{2} \mathrm{~S}_{2} \mathrm{O}_{3}$ (about $10 \mathrm{ml}$ ) and water. The mixture was extracted with ethyl acetate $(10 \mathrm{ml} \times 4)$ and the combined extracts were washed with saturated aq $\mathrm{NaCl}$ and dried over anhydrous $\mathrm{Na}_{2} \mathrm{SO}_{4}$. Evaporation $(1 \mathrm{mmHg}, 24 \mathrm{~h}, 1 \mathrm{mmHg}=133.322 \mathrm{~Pa})$ afforded 3-(2-oxo-2-phenylethyl)cyclohexane-1,2-dione (2a) (203.3 $\mathrm{mg}, 0.883 \mathrm{mmol}, 89 \%)$.

The spectral data of product $\mathbf{2 a}$ are as follows:

Yellow crystals; $\mathrm{mp}$ (decomp) $86.5-88.5^{\circ} \mathrm{C}$ (chloroform and hexane); IR (KBr) 1714, 1689, 1448, 1371, 1357, 1244, 1219, 1001, 766, 731, $694 \mathrm{~cm}^{-1}$; ${ }^{1} \mathrm{H}$ NMR $\delta=1.82-1.90(1 \mathrm{H}, \mathrm{m}(\mathrm{dq}-$ like)), $2.00-2.10(1 \mathrm{H}, \mathrm{m}$ (q-like) ), 2.17-2.26 (2H, m), $2.68(1 \mathrm{H}$, ddd, $J=6.3,13.6,16.9 \mathrm{~Hz}), 2.95-2.99(1 \mathrm{H}, \mathrm{m}$ (ddd-like)), 3.17$3.21(1 \mathrm{H}, \mathrm{m}), 3.29(1 \mathrm{H}, \mathrm{dd}, J=6.3,8.1 \mathrm{~Hz}), 3.62(1 \mathrm{H}, \mathrm{dd}, J=$ 3.9, 8.1 Hz), 7.44-7.48 $(2 \mathrm{H}, \mathrm{m}(\mathrm{t}-\mathrm{like})), 7.56-7.59(1 \mathrm{H}, \mathrm{m}(\mathrm{t}-$ like)) $7.95(2 \mathrm{H}, \mathrm{dd}, J=1.3,8.4 \mathrm{~Hz}) ;{ }^{13} \mathrm{CNMR}\left(\mathrm{CD}_{2} \mathrm{Cl}_{2}\right) \delta=22.91$, $30.27,39.46,43.21,48.82,128.12,128.23,128.76,128.83,133.57$, 136.62, 197.28, 198.27, 199.75. HRMS: $\mathrm{m} / \mathrm{z}$ 230.0947. Calcd for $\mathrm{C}_{14} \mathrm{H}_{14} \mathrm{O}_{3}: \mathrm{M}, 230.0943$.

Typical Procedure to Prepare 4,5-Dihydro-7(6H)-benzofuranone Derivative from Cyclohexane-1,2-dione. To a solution of CAN $(1.156 \mathrm{~g}, 2.11 \mathrm{mmol})$ in acetonitrile $(5.0 \mathrm{ml})$ was added a solution of trimethyl(1-phenylvinyloxy)silane (4a) $(579.6 \mathrm{mg}, 3.01$ $\mathrm{mmol})$ and cyclohexane-1,2-dione $(\mathbf{1})(112.0 \mathrm{mg}, 0.999 \mathrm{mmol})$ in acetonitrile $(5.0 \mathrm{ml})$ at $-35^{\circ} \mathrm{C}$ under an argon atmosphere. After $15 \mathrm{~min}$, the reaction was quenched by adding $0.1 \mathrm{~mol} \mathrm{dm}^{-3}$ aq $\mathrm{Na}_{2} \mathrm{~S}_{2} \mathrm{O}_{3}$ (about $10 \mathrm{ml}$ ) and water. The mixture was extracted with ethyl acetate $(15 \mathrm{ml} \times 4)$ and the combined extracts were washed with saturated aq $\mathrm{NaCl}$ and dried over anhydrous $\mathrm{Na}_{2} \mathrm{SO}_{4}$. After the evaporation of the solvent, Amberlyst $15(80 \mathrm{mg})$ was added to a solution of the crude triketone $2 \mathbf{a}$ in toluene $(40 \mathrm{ml})$. The reaction mixture was refluxed at $16 \mathrm{~h}$, and then Amberlyst 15 was removed by filtration. After the evaporation, aluminum column chromatographic purification afforded 2-phenyl-4,5-dihydro-7(6H)benzofuranone (3a) (165.7 mg, $0.781 \mathrm{mmol}, 78 \%$ ).

The spectral data of the products are as follows:

2-Phenyl-4,5-dihydro-7(6H)-benzofuranone (3a). Colorless crystals; mp 81.5-82.0 ${ }^{\circ} \mathrm{C}$ (chloroform); IR (KBr) 1664, 1475, $1454,1431,1410,1138,926,897 \mathrm{~cm}^{-1} ;{ }^{1} \mathrm{H}$ NMR $\delta=2.13-2.18$ $(2 \mathrm{H}, \mathrm{m}), 2.58(2 \mathrm{H}, \mathrm{t}, J=6.4 \mathrm{~Hz}), 2.78(2 \mathrm{H}, \mathrm{t}, J=6.1 \mathrm{~Hz}), 6.65(1 \mathrm{H}, \mathrm{s})$, $7.34-7.41$ (3H, m), 7.78-7.80 (2H, dd, $J=1.2,8.4 \mathrm{~Hz}),{ }^{13} \mathrm{C} \mathrm{NMR}$ $\delta=23.10,24.32,38.28,106.50,125.36,128.83,129.22,129.46$, 142.03, 147.00, 158.54, 185.66. Found: C, 79.24, H, 5.65\%. Calcd for $\mathrm{C}_{14} \mathrm{H}_{12} \mathrm{O}_{2}$ : C, $79.23 ; \mathrm{H}, 5.70 \%$.

3-Methyl-2-phenyl-4,5-dihydro-7(6H)-benzofuranone (3b). Colorless crystals; mp 154.5-155.0 ${ }^{\circ} \mathrm{C}$ (chloroform); IR (KBr) $1660,1456,1431,1387,1362,1336,1109,897,775,698 \mathrm{~cm}^{-1}$; ${ }^{1} \mathrm{H}$ NMR $\delta=2.14-2.19(2 \mathrm{H}, \mathrm{m}$ (tt-like)), $2.22(3 \mathrm{H}, \mathrm{s}) 2.57$ (2H, t, $J=$ $6.4 \mathrm{~Hz}), 2.69(2 \mathrm{H}, \mathrm{t}, J=6.1 \mathrm{~Hz}), 7.33-7.36(1 \mathrm{H}, \mathrm{m}(\mathrm{t}-\mathrm{like})), 7.41-$ $7.44(2 \mathrm{H}, \mathrm{m}$ (t-like) $), 7.74-7.76(2 \mathrm{H}, \mathrm{m}(\mathrm{d}-$ like $)) ;{ }^{13} \mathrm{CNMR} \delta=$
9.81, 21.59, 24.11, 38.24, 116.71, 126.75, 128.62, 128.70, 130.24, 142.62, 145.67, 154.06, 185.76. Found: C, 79.51; H, 6.29\%. Calcd for $\mathrm{C}_{15} \mathrm{H}_{14} \mathrm{O}_{2}: \mathrm{C}, 79.62 ; \mathrm{H}, 6.24 \%$.

2-(2-Phenylethyl)-4,5-dihydro-7(6H)-benzofuranone (3c-1) and 3-Benzyl-2-methyl-4,5-dihydro-7 $(6 \mathrm{H})$-benzofuranone (3c2). These compounds were obtained as an inseparable mixture.

Colorless oil; IR (neat) 2945, 1670, 1529, 1448, 1437, 1417, $1132,895,733,702 \mathrm{~cm}^{-1} ;{ }^{13} \mathrm{C}$ NMR $\delta=12.21,21.77,22.95,24.11$, $24.32,29.39,30.16,33.71,37.79,37.97,108.02,119.22,126.25$, $126.34,128.04,128.21,128.44,128.56,139.00,140.32,141.62$, $141.67,145.80,146.63,155.48,161.73,185.38,185.52$. (3c-1) ${ }^{1} \mathrm{H} N M R \quad \delta=2.07-2.12(2 \mathrm{H}, \mathrm{m}), 2.07-2.12(2 \mathrm{H}, \mathrm{m}), 2.51-2.53$ $(2 \mathrm{H}, \mathrm{m}(\mathrm{t}-\mathrm{like})), 2.68(2 \mathrm{H}, \mathrm{t}, J=6.1 \mathrm{~Hz}), 2.98(4 \mathrm{H}, \mathrm{s}), 5.99(1 \mathrm{H}$, s), $7.10-7.28(5 \mathrm{H}, \mathrm{m}) .(3 \mathrm{c}-2){ }^{1} \mathrm{H}$ NMR $\delta=2.01-2.06(2 \mathrm{H}, \mathrm{m})$, $2.32(3 \mathrm{H}, \mathrm{s}), 2.43-2.48(4 \mathrm{H}, \mathrm{m}), 3.70(2 \mathrm{H}, \mathrm{s}), 7.10-7.28(5 \mathrm{H}, \mathrm{m})$. HRMS: $m / z$ 240.1154. Calcd for $\mathrm{C}_{16} \mathrm{H}_{16} \mathrm{O}_{2}$ : M, 240.1150.

2-Isopropyl-4,5-dihydro-7(6H)-benzofuranone (3d). Colorless oil; IR (neat) 2968, 2937, 2873, 1672, 1527, 1437, 1327, 1136, $1068,958,897,594 \mathrm{~cm}^{-1} ;{ }^{1} \mathrm{HNMR} \delta=1.25(3 \mathrm{H}, \mathrm{s}), 1.27(3 \mathrm{H}$, s), $2.07-2.12(2 \mathrm{H}, \mathrm{m}), 2.51(2 \mathrm{H}, \mathrm{t}, J=6.5 \mathrm{~Hz}), 2.69(2 \mathrm{H}, \mathrm{t}, J=6.1$ $\mathrm{Hz}), 2.95-3.00(1 \mathrm{H}, \mathrm{m}), 6.02(1 \mathrm{H}, \mathrm{s}) ;{ }^{13} \mathrm{CNMR} \delta=20.80,20.80$, 23.06, 24.38, 28.24, 38.06, 105.49, 141.61, 146.41, 168.15, 185.48 . HRMS: $m / z$ 178.0978. Calcd for $\mathrm{C}_{11} \mathrm{H}_{14} \mathrm{O}_{2}$ : $\mathrm{M}, 178.09942$.

1,2,6,7,8,9-Hexahydro-4(3H)-dibenzofuranone (3e). Colorless crystals; mp $60.0-61.0^{\circ} \mathrm{C}$ (chloroform); IR (neat) 2939, $1741,1668,1543,1468,1439,1346,1130,949 \mathrm{~cm}^{-1} ;{ }^{1} \mathrm{HNMR}$ $\delta=1.71-1.76(2 \mathrm{H}, \mathrm{m}), 1.81-1.86(2 \mathrm{H}, \mathrm{m}), 2.07-2.12(2 \mathrm{H}, \mathrm{m})$, $2.34-2.37(2 \mathrm{H}, \mathrm{m}(\mathrm{t}-$ like $)), 2.50(2 \mathrm{H}, \mathrm{t}, J=6.4 \mathrm{~Hz}), 2.60(2 \mathrm{H}, \mathrm{t}$, $J=6.1 \mathrm{~Hz}), 2.63(2 \mathrm{H}, \mathrm{t}, J=6.3 \mathrm{~Hz}) ;{ }^{13} \mathrm{CNMR} \delta=20.36,21.55$, $22.45,22.47,23.54,24.27,38.02,118.56,140.51,145.86,158.12$, 185.44. Found: $\mathrm{C}, 75.53 ; \mathrm{H}, 7.34$. Calcd for $\mathrm{C}_{12} \mathrm{H}_{14} \mathrm{O}_{2}$ : C, 75.76; H, $7.42 \%$.

4,5-Dihydro-7(6H)-benzofuranone (3f). Colorless crystals; mp 59.0-59.5 ${ }^{\circ} \mathrm{C}$ (petroleum ether) (lit, ${ }^{2 a)} \mathrm{mp} 60-61{ }^{\circ} \mathrm{C}$ (petroleum ether)); IR (KBr) 3122, 2943, 1589, 1475, 1434, 1410, $1304,1113,887,814 \mathrm{~cm}^{-1} ;{ }^{1} \mathrm{H} N M R \quad \delta=2.11-2.16(2 \mathrm{H}, \mathrm{m}), 2.55$ $(2 \mathrm{H}, \mathrm{t}, J=6.0 \mathrm{~Hz}), 2.75(2 \mathrm{H}, \mathrm{t}, J=6.1 \mathrm{~Hz}), 6.39(1 \mathrm{H}, \mathrm{d}, J=1.7 \mathrm{~Hz})$, $7.54(1 \mathrm{H}, \mathrm{d}, J=1.7 \mathrm{~Hz})$.

7a-Hydroxy-2-methyl-2-phenylperhydrobenzofuran-7-one (3h). Though two diastereomers $(\mathrm{A}: \mathrm{B}=7: 3)$ were isolated by preparative TLC, their relative configurations are not determined.

Diastereomer A. Colorless crystals; $\mathrm{mp} 81.0-82.0^{\circ} \mathrm{C}$ (hexane); IR (KBr) 3456, 2962, 2935, 1722, 1448, 1407, 1115, 1080, $1061,1028,1005,955,700 \mathrm{~cm}^{-1} ;{ }^{1} \mathrm{H}$ NMR $\delta=1.14-1.24(1 \mathrm{H}$, $\mathrm{m}), 1.50-1.59(2 \mathrm{H}, \mathrm{m}), 1.64(3 \mathrm{H}, \mathrm{s}), 1.81-1.83(1 \mathrm{H}, \mathrm{m}), 2.34$ $(1 \mathrm{H}, \mathrm{d}, J=12.3 \mathrm{~Hz}), 2.44-2.53(2 \mathrm{H}, \mathrm{m}), 2.62-2.70(2 \mathrm{H}, \mathrm{m})$, $4.60(1 \mathrm{H}, \mathrm{s}), 7.19-7.22(1 \mathrm{H}, \mathrm{m}(\mathrm{t}-$ like $)), 7.30-7.33(2 \mathrm{H}, \mathrm{m}(\mathrm{t}-$ like)), 7.39-7.41 (2H, m (d-like)); ${ }^{13} \mathrm{CNMR} \delta=24.61,28.27$, $35.09,37.17,44.72,49.61,87.00,101.58,124.33,126.26,128.23$, 149.38, 206.42. Found: C, 72.94; $\mathrm{H}, 7.33 \%$. Calcd for $\mathrm{C}_{15} \mathrm{H}_{18} \mathrm{O}_{3}$ : C, $73.15 ; \mathrm{H}, 7.37 \%$.

Diastereomer B. Colorless oil; IR (neat) 3481, 2970, 2947, 1726, 1448, 1115, 1074, 1036, 1005, 760, $704 \mathrm{~cm}^{-1} ;{ }^{1} \mathrm{HNMR} \delta=$ $1.65(3 \mathrm{H}, \mathrm{s}), 1.69-1.82(2 \mathrm{H}, \mathrm{m}), 1.84-1.90(1 \mathrm{H}, \mathrm{m}), 2.03-2.10$ $(1 \mathrm{H}, \mathrm{m}), 2.19(1 \mathrm{H}, \mathrm{dd}, J=1.0,12.5 \mathrm{~Hz}), 2.48-2.56(2 \mathrm{H}, \mathrm{m}), 2.72-$ $2.77(1 \mathrm{H}, \mathrm{m}), 2.80(1 \mathrm{H}, \mathrm{dd}, J=7.6,12.5 \mathrm{~Hz}), 4.16(1 \mathrm{H}, \mathrm{s}), 7.15-$ $7.18(1 \mathrm{H}, \mathrm{m}$ (t-like)), 7.25-7.29 (2H, m (t-like)), 7.34-7.36 (2H, $\mathrm{m}$ (d-like)); ${ }^{13} \mathrm{C}$ NMR $\delta=24.32,29.67,33.77,37.27,44.12,48.83$, $86.66,102.00,124.41,126.20,127.82,150.78,206.46$. HRMS Found: $m / z$ 228.1150. Calcd for $\mathrm{C}_{15} \mathrm{H}_{16} \mathrm{O}_{2}: \mathrm{M}-\mathrm{H}_{2} \mathrm{O}, 228.1150$.

3-(2-Oxo-2-phenylethyl)cyclopentane-1,2-dione (7). Yellow crystals; $\mathrm{mp}$ (decomp) $124.0-126.0{ }^{\circ} \mathrm{C}$ (ether and petroleum 
ether); IR (KBr) 3051, 2976, 1718, 1689, 1444, 1253, 1219, 758, $696 \mathrm{~cm}^{-1} ;{ }^{1} \mathrm{HNMR} \delta=1.84-2.21(2 \mathrm{H}, \mathrm{m}), 2.32-2.44(2 \mathrm{H}, \mathrm{m})$, $2.94-3.01(1 \mathrm{H}, \mathrm{m}), 3.35(1 \mathrm{H}, \mathrm{dd}, J=4.7,17.7 \mathrm{~Hz}), 3.45(1 \mathrm{H}, \mathrm{dd}$, $J=8.7,17.7 \mathrm{~Hz}), 7.46-7.49(2 \mathrm{H}, \mathrm{m}(\mathrm{t}-\mathrm{like})), 7.55-7.60(1 \mathrm{H}, \mathrm{m}(\mathrm{t}-$ like)) $7.95-7.96\left(2 \mathrm{H}, \mathrm{m}\right.$ (d-like)); ${ }^{13} \mathrm{CNMR}\left(\mathrm{CD}_{2} \mathrm{Cl}_{2}\right.$ and DMSO$\left.d_{6}\right) \delta=27.17,31.84,39.76,40.53,128.05,128.67,133.17,136.96$, $174.79,176.56,198.15$. HRMS Found: $\mathrm{m} / \mathrm{z}$ 216.0789. Calcd for $\mathrm{C}_{14} \mathrm{H}_{14} \mathrm{O}_{3}: \mathrm{M}, 216.0786$.

3-(2-Oxo-2-phenylethyl)cycloheptane-1,2-dione (8). Yellow crystals; $\mathrm{mp}$ (decomp) $82.0-83.0^{\circ} \mathrm{C}$ (ether); IR (KBr) 2931, 1716, $1678,1448,1363,1232,1205,766,692 \mathrm{~cm}^{-1} ;{ }^{1} \mathrm{HNMR} \delta=1.55$ $1.74(3 \mathrm{H}, \mathrm{m}), 2.00-2.19(3 \mathrm{H}, \mathrm{m}), 2.61-2.67(1 \mathrm{H}, \mathrm{m}), 2.70-2.79$ $(1 \mathrm{H}, \mathrm{m}), 3.07(1 \mathrm{H}, \mathrm{dd}, J=5.6,18.0 \mathrm{~Hz}), 3.37-3.45(1 \mathrm{H}, \mathrm{m}), 3.65$ $(1 \mathrm{H}, \mathrm{dd}, J=7.2,18.0 \mathrm{~Hz}), 7.43-7.46(2 \mathrm{H}, \mathrm{m}$ (t-like) $), 7.54-7.57$ $\left(1 \mathrm{H}, \mathrm{m}\right.$ (t-like)), 7.95-7.96 (2H, m (d-like)); ${ }^{13} \mathrm{C}$ NMR $\delta=24.23$, 28.72, 32.61, 39.42, 40.18, 45.08, 128.11, 128.62, 133.38, 136.32, 197.16, 203.81, 205.18. HRMS Found: $\mathrm{m} / \mathrm{z} 244.1104$. Calcd for $\mathrm{C}_{14} \mathrm{H}_{14} \mathrm{O}_{3}: \mathrm{M}, 244.1099$.

2-Phenyl-5,6,7,8-tetrahydro- $4 \mathrm{H}$-cyclohepta[b]furan-8-one (10). Colorless crystals; mp 95.0-95.5 ${ }^{\circ} \mathrm{C}$ (chloroform); IR (KBr) 2925, 1635, 1479, 1450, 1412, 1348, 1267, 1092, 930, 806, $769,692 \mathrm{~cm}^{-1} ;{ }^{1} \mathrm{HNMR} \delta=1.88-1.99(4 \mathrm{H}, \mathrm{m}), 2.73(2 \mathrm{H}, \mathrm{t}, J=6.1$ Hz), $2.86(2 \mathrm{H}, \mathrm{m}$ (t-like)), $6.58(1 \mathrm{H}, \mathrm{s}), 7.31-7.41(3 \mathrm{H}, \mathrm{m}), 7.77-$ 7.79 (2H, m (d-like)); ${ }^{13} \mathrm{C}$ NMR $\delta=22.49,26.20,27.21,42.32$, 109.28, 125.24, 128.71, 128.71, 129.19, 137.47, 148.07, 157.36, 189.41. Found: C, 79.32; $\mathrm{H}, 6.20 \%$. Calcd for $\mathrm{C}_{15} \mathrm{H}_{14} \mathrm{O}_{2}$ : C, $79.62 ; \mathrm{H}, 6.24 \%$.

This work was supported by a Grant-in-Aid for Scientific Research on Priority Area No. 08245101 from the Ministry of Education, Science, Sports and Culture.

\section{References}

1) a) H. Richter and G. Spiteller, Chem. Ber, 112, 1088 (1979); b) J. Endo, Yakugaku Zasshi, 95, 1321 (1975).

2) a) E. J. Walsh, Jr., and G. B. Stone, Tetrahedron Lett., 27, 1127 (1986); b) E. J. Corey and Y. B. Xiang, Tetrahedron Lett., 28, 5403 (1987).

3) A. A. Ponaras, Tetrahedron Lett., 21, 4803 (1980).

4) M. Utaka, H. Kuriki, T. Sakai, and A. Takeda, J. Org. Chem., 51, 935 (1986).

5) a) K. Narasaka, N. Miyoshi, K. Iwakura, and T. Okauchi, Chem. Lett., 1989, 2169; b) K. Narasaka, T. Okauchi, K. Tanaka, and M. Murakami, Chem. Lett., 1992, 2099; c) K. Narasaka and H. Sakurai, Chem. Lett., 1993, 1269; d) N. Iwasawa, S. Hayakawa, M. Funahashi, K. Isobe, and K. Narasaka, Bull. Chem. Soc. Jpn., 66, 819 (1993); e) Y. Kohno and K. Narasaka, Bull. Chem. Soc. Jpn., 68, 322 (1995); f) N. Arai and K. Narasaka, Bull. Chem. Soc. Jpn., 70, 2525 (1997).

6) E. I. Heiba and R. M. Dessau, J. Org. Chem., 39, 3456 (1974).

7) E. Baciocchi and R. Ruzziconi, Synth. Commun., 18, 1841 (1988).

8) D. V. Rao, F. A. Stuber, and H. Ulrich, J. Org. Chem., 44, 456 (1979).

9) D. N. Hendrickson, Y. S. Sohn, and H. B. Gray, Inorg. Chem., 10, 1559 (1971).

10) M. M. Ray, J. N. Adhya, D. Biswas, and S. N. Poddar, Aust. J. Chem., 19, 1737 (1966).

11) R. W. Vander, R. C. Voter, and C. V. Banks, J. Org. Chem., 14, 836 (1949).

12) T. Mukaiyama and K. Narasaka, Org. Synth., 65, 6 (1987).

13) R. F. Cunico and C. P. Kuan, J. Org. Chem., 50, 5410 (1985).

14) M. S. Newman and M. C. Vander Zwan, J. Org. Chem., 38, 2910 (1973). 